\title{
ALL MAXIMAL COMPLETELY REGULAR SUBMONOIDS OF $\operatorname{Hyp}_{G}(2)$
}

\author{
Pornpimol KunAma
}

AND

\author{
SORASAK LEERATANAVALEE ${ }^{1}$ \\ Department of Mathematics \\ Faculty of Science, Chiang Mai University \\ Chiang Mai 50200, Thailand \\ e-mail: pornpimol5331@gmail.com \\ sorasak.1@cmu.ac.th
}

\begin{abstract}
In this paper we consider mappings $\sigma$ which map the binary operation symbol $f$ to the term $\sigma(f)$ which do not necessarily preserve the arity. These mapping are called generalized hypersubstitutions of type $\tau=(2)$ and we denote the set of all these generalized hypersubstitutions of type $\tau=(2)$ by $\operatorname{Hyp}_{G}(2)$. The set $\operatorname{Hyp}_{G}(2)$ together with a binary operation defined on this set and the identity generalized hypersubstitution which maps $f$ to the term $f\left(x_{1}, x_{2}\right)$ forms a monoid. In this paper, we determine all maximal completely regular submonoids of this monoid.
\end{abstract}

Keywords: generalized hypersubstitution, regular element, completely regular.

2010 Mathematics Subject Classification: 20M07, 08B15, $08 \mathrm{~B} 25$.

\section{REFERENCES}

[1] A. Boonmee and S. Leeratanavalee, All completely regular elements in Hyp $(n)$, Discuss. Math. Gen. Alg. and Appl. 33 (2013) 211-219. doi:10.7151/DMGAA.1203

[2] K. Denecke, D. Lau, R. Pöschel and D. Schweigert, Hyperidentities, hyperequational classes and clone congruences, Contributions to General Algebra 7 (Verlag HolderPichler-Tempsky, Wein, 1991) 97-118.

\footnotetext{
${ }^{1}$ Corresponding author.
} 
[3] W. Puninagool and S. Leeratanavalee, All regular elements in $H y p_{G}(2)$, Kyungpook Math. J. 51 (2011) 139-143.

doi:10.5666/KMJ.2011.51.2.139

[4] W. Puninagool and S. Leeratanavalee, The monoid of generalized hypersubstitutions of type $\tau=(n)$, Discuss. Math. Gen. Alg. and Appl. 30 (2010) 173-191.

[5] J.M. Howie, Fundamentals of Semigroup Theory (Academic Press, London, 1995).

[6] M. Petrich and N.R. Reilly, Completely Regular Semigroups (John Wiley and Sons, Inc., New York, 1999).

[7] S. Leeratanavalee and K. Denecke, Generalized Hypersubstitutions and Strongly Solid Varieties, General Algebra and Applications, Proc. of the "59 th Workshop on General Algebra, "15 th Conference for Young Algebraists Potsdam 2000" (Shaker Verlag, 2000) 135-145.

Received 29 March 2017

Revised 2 April 2017 